\title{
Influence de l'incorporation de saindoux dans I'aliment concentré sur la croissance et les qualités de carcasse chez l'agneau mâle sevré précocement
}

\author{
G Bozzolo 1, M Bouillier-Oudot 1, P Quenardelle 1, M Candau 1
}

avec la collaboration technique de D Grasset ${ }^{2}$ et $\mathrm{H}_{\text {Manse }}{ }^{1}$

${ }^{1}$ ENSAT, École nationale supérieure agronomique, 31076 Toulouse cedex;

2 GEBRO, Groupement des éleveurs de brebis du bassin de Roquefort, 12001 Millau cedex, France

(Reçu le 27 octobre 1991; accepté le 13 février 1992)

\begin{abstract}
Résumé - L'effet de l'addition de 3\% de saindoux dans l'aliment d'engraissement est analysé sur la croissance, l'état des profils fermentaires dans le rumen et les caractéristiques de carcasse (conformation, rendement, importance et qualité de l'état d'engraissement) d'agneaux mâles Lacaune. L'aliment comprenant du saindoux (ALS) a été comparé à un témoin (ALT) isoénergétique (1 UFV/kg $\mathrm{MS}$ ) et iso-azoté (160 g MAT/kg MS). Ils ont été offerts à volonté, après distribution d'un aliment commun de démarrage durant $22 \mathrm{j}$. Les animaux, âgés de $28 \mathrm{j}$ et pesant $12 \mathrm{~kg}$ en moyenne au sevrage, ont été abattus au poids vif moyen de $40 \mathrm{~kg}$. La comparaison des caractéristiques de croissance et de carcasse a été réalisée à partir d'un échantillon normalisé ( 80 agneaux/lot) sur la base du gain moyen quotidien entre 0 et $20 \mathrm{j}$ d'engraissement $\left(G M Q_{0-20 \mathrm{j}}=146 \mathrm{~g} / \mathrm{j} ; \mathrm{cv}=52 \%\right)$. L'évolution des profils fermentaires du rumen a été suivie à partir de prélèvements effectués à 20 , à 50 j d'engraissement et à l'abattage sur 6 agneaux par lot, et en fermenteurs semi-continus (Rusitec). Les vitesses de croissance ne diffèrent pas entre les 2 lots sur l'ensemble de l'engraissement (GMQ $Q_{0-A b a}$ $\left.=310 \mathrm{~g} / \mathrm{j} ; G M Q_{20-A b a}=361 \mathrm{~g} / \mathrm{j}\right)$. Alors que les critères de rendement carcasse, de conformation et d'état d'engraissement ne diffèrent pas, la qualité des gras externes est améliorée par l'apport de saindoux $(P<0.05)$. La proportion de carcasses fermes est supérieure dans ce cas $(61,3$ vs $46,3 \%$ pour ALT); les carcasses de couleur blanche sont plus nombreuses (50 vs $35 \%$ ). La présence de saindoux ne perturbe pas l'évolution des facies fermentaires dans le rumen. Enfin, les indices de consommation ne different pas sur l'ensemble de l'engraissement $\left(l_{0-A b a}=3,49\right)$. L'absence d'effet néfaste sur les performances de croissance et sur l'efficacité alimentaire permet de penser qu'une augmentation du taux d'incorporation est envisageable pour renforcer l'effet bénéfique constaté sur la qualité des carcasses.
\end{abstract}

agneau mâle / saindoux / croissance / qualité des carcasses

Summary - Influence of lard incorporation in the fattening diet upon growth and carcass quality of early weaned male lambs. The influence of $3 \%$ lard incorporation in the fattening diet was investigated on growth parameters, rumen fermentation (VFA balance) and carcass characteristics of early weaned male lambs, intensively fattened in the pen during winter. Lacaune lambs $(12 \mathrm{~kg}$ 
live weight and $28 \mathrm{~d}$ old at weaning) were randomized in 2 groups and fed the same starter pelleted diet for 22 days. The fattening diet was then provided consisting of 3\% lard (ALS) and compared with a reference diet $(A L T)$, with the following isoenergetic and isoproteic values: ME: $2.77 \mathrm{Mcal} ; \mathrm{CP}: 16 \%$ $D M$. Ratios of alimentary fats were $5.5 \%$ for ALS and $3.2 \%$ of DM for ALT. The stearic and palmitic percentages were respectively $0.54 \%$ and $1.23 \%$ vs $0.23 \%$ and $0.67 \%$. The animals were slaughtered at $40 \mathrm{~kg}=$ live weight. Comparisons of growth and carcass characteristics were made with a normalised sample (80 lambs per group) based on their ADG between 0 and $20 d$ with the same starter $\operatorname{diet}\left(\mathrm{ADG}_{\mathrm{O}-20 \mathrm{~d}}=146 \mathrm{~g} / \mathrm{d} ; \mathrm{cv}=52 \%\right)$. The rumen fermentary balance (NFA\%) was followed up from 3 samples taken with an esophageal cannula (at day 20,40 and at slaughter) in 6 lambs per diet and with semi-continuous incubators (Rusitec) during the transition period between the post-weaning and fattening diets. The average growth rate did not differ between the 2 groups during the entire fattening period $\left(A D G_{O-A b a}=310 \mathrm{~g} / \mathrm{d} ; \mathrm{ADG}_{20-A b a}=361 \mathrm{~g} / \mathrm{d}\right)$. The dressing percentage, conformation and state for fattening criteria were not different, but lard significantly $(P<0.05)$ improved the external fat carcass quality. The firm carcass ratio was higher in this case ( 61.3 vs $46.3 \%$ for the reference diet); likewise, white carcasses were more numerous (50 vs $35 \%$ ). The presence of lard in the diet does not alter the fermentary balance in the rumen. During the entire fattening period, VFA rates evolve in both groups until they attain the acetic, propionic and butyric acid percentages of 52, 35 and 7 respectively. Finally, feed conversion ratios do not differ over the total growing period $\left(\mathrm{lc}_{O-A b a}=3.49 \mathrm{~kg} \mathrm{DM} / \mathrm{kg}\right.$ live gain). The lack of negative influence on growth scores and alimentary efficiency lead us to believe that higher ratios of lard in the diet will possibly increase carcass quality.

ram lamb / lard / growth / carcass quality

\section{INTRODUCTION}

Le rythme de croissance des agneaux de bergerie dépend de l'origine génétique des animaux mais aussi de leur régime alimentaire, élément essentiel de la conduite d'élevage (Ørskov et al, 1975; Busboom et al, 1981; Turgeon et al, 1986; Aurousseau, 1986). Les qualités de la carcasse intègrent, en plus des aspects technologiques classiques (poids et conformation de la carcasse, état d'engraissement), des critères de nature plus subjective comme la tenue et la couleur des gras de carcasse après ressuyage et réfrigération.

L'apparition de graisses molles, pour la plupart colorées, est un phénomène complexe. Celle-ci est souvent associée aux régimes d'engraissement à forte concentration énergétique d'origine céréalière, par nature propiogéniques (Garton et al, 1972; Molénat et Thériez, 1973; Ørskov et al, 1975; Field et al, 1978; Miller et al, 1980; Aurousseau, 1981). Ces rations aboutissent à la formation et au dépôt de triglycérides à bas point de fusion, riches en acides gras saturés de type ramifié et en acides gras insaturés, par ailleurs sensibles à l'oxydation durant la conservation des carcasses (l'Estrange et Mulvihill, 1975; Bremmer et al, 1976; Palmer et al, 1986; Faustman et al, 1989).

L'évidence d'une interaction entre rythme de croissance et qualité de carcasse (Aurousseau et al, 1973; Crouse et al, 1978) notamment chez l'agneau de Roquefort (Bozzolo et al, 1990), nous a conduit à analyser les effets directs ou indirects de l'incorporation de lipides alimentaires sur les rythmes de croissance et la qualité des graisses produites. Aurousseau et al (1973), chez l'agneau préruminant, mentionnaient l'incidence rémanente des corps gras introduits dans l'aliment d'allaitement. Bozzolo et al (1991) ont confirmé l'influence positive de l'huile de coprah incorporée dans le régime de transition d'agneaux Lacaune néorumi- 
nants, sevrés précocement, surtout pour les plus légers d'entre eux au sevrage. Bien que l'apport fut limité dans le temps $(20 \mathrm{j})$, cette influence se reportait 2 mois plus tard sur la qualité de l'engraissement. Or, le rythme d'accrétion des lipides dans le processus d'adipogénèse s'accroît dès que l'agneau dépasse environ $30 \%$ de son poids de maturité (Andrews et Ørskov, 1970; Vezinhet et al, 1976; Robelin et al, 1977; Thériez et al, 1981; Thompson et Parks, 1985). Si les acides gras à chaîne moyenne apportés par l'huile de coprah, en période de transition, peuvent avoir une influence bénéfique sur le rythme de croissance en raison de leur disponibilité énergétique immédiate dans cette situation de précarité énergétique (Cera et al, 1989; Aurousseau et al, 1989; Mahan, 1991), il en va différemment pendant la phase de finition. À ce stade, l'approvisionnement énergétique n'est plus limitant mais est plutôt excédentaire pour des animaux nourris ad libitum. Sa maîtrise reste donc une clef essentielle pour guider la constitution et la qualité des tissus de réserve.

Compte tenu de la particularité des ruminants à synthétiser la majorité des triglycérides dans la cellule adipeuse à partir d'acides gras produits par la synthèse de novo intra-adipocytaire et/ou issus de la lipolyse des triglycérides circulants (Christie, 1981; Annisson, 1984), la nature des acides gras alimentaires peut influencer directement la qualité des graisses de l'agneau, durant cette période de finition. Ces acides gras alimentaires sont en grande proportion saturés à cause de l'hydrogénation réalisée dans le rumen de ce ruminant désormais accompli. Ceux-ci pourraient donc équilibrer la production endogène (de novo) d'acides gras, en majorité insaturés (Ray et al, 1975), pour peu que leur digestibilité soit bonne et que la capacité d'hydrogénation de la flore du rumen ne soit pas réduite dans le cas des AG alimentaires longs et insaturés (Miller et Rice, 1967; Cameron et Hogue, 1968; Bauchart et al, 1985). Pour les AG saturés, plusieurs inconvénients peuvent apparaître : l'incorporation de graisses alimentaires saturées a pour effet de réduire l'appétit des animaux (Kowalczyk et al, 1977; Sutton et al, 1983), de diminuer leur digestibilité par la formation de savons (Devendra et Lewis, 1974; Jenkins et Palmquist, 1982; Jenkins et Jenny, 1989; Bock et al, 1991) mais aussi de conduire à des triglycérides à plus bas point de fusion en raison de l'activité des désaturases tissulaires s'exerçant notamment sur l'acide stéarique (Wahle et Garton, 1972; Christie, 1981).

Par ailleurs, Crouse et al (1978) invoquent que les régimes à forte concentration énergétique induisent, à poids constant, une maturité plus précoce des agneaux. Chez les mâles de race Lacaune, les individus dotés d'une forte vitesse de croissance sont, à poids constant, moins précoces (anabolisme protidique plus élevé, degré d'engraissement moindre) et sont candidats à générer des carcasses aux graisses molles (Bozzolo et al, 1990). Dans ces conditions, l'incidence d'une supplémentation en acides gras longs saturés, à partir d'une source facilement digestible comme c'est le cas du saindoux (Thieulin, 1968) paraissait intéressante à considérer. En effet, I'hydrolyse des triglycérides dans le rumen peut-être plus ou moins prononcée selon leur origine (de 95 à $35 \%$ ) et reste plus faible pour les graisses animales (Church, 1979). Ce travail rapporte les effets observés d'une telle incorporation dans l'aliment de croissancefinition, tant sur les performances de croissance et l'efficacité alimentaire que sur la qualité des carcasses produites. 


\section{MATÉRIEL ET MÉTHODES}

\section{Animaux}

Cet essai alimentaire a été réalisé avec des agneaux mâles de race Lacaune (rameau laitier), sevrés précocement (entre 4 et 5 semaines). Ceux-ci ont été nourris en bergerie, en hiver-printemps et abattus sur la base d'un poids constant $(40 \mathrm{~kg}$ ) après un tri hebdomadaire. Au total 230 agneaux, répartis en 2 lots, dans la même bergerie, ont été contrôlés individuellement. Les animaux mis en comparaison sur les caractéristiques de croissance et de carcasse ont été sélectionnés pour réaliser des échantillons normalisés et de sorte à respecter au mieux des conditions de départ identiques. C'est sur la base du gain moyen quotidien entre 0 et 20 j d'engraissement (GMQ0-20) qu'a été constitué l'échantillonnage (fig 1). Nous avons considéré cet indicateur comme une mesure indirecte de la variabilité des effets non maîtrisés (génétique, stress, adaptation) durant la période de transition alors que les agneaux étaient soumis à un même régime alimentaire. En effet, les conditions d'application d'un traitement par covariance n'étaient pas remplies. La standardisation a été réalisée en retenant pour contraintes

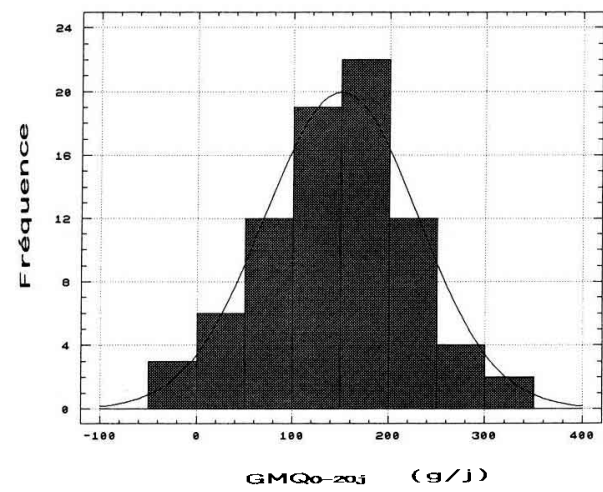

Fig 1. Composition des échantillons d'agneaux comparés : histogramme des fréquences après standardisation des échantillons respectifs : témoin et saindoux, sur la base des GMQ entre 0 et 20 jours - superposition de la courbe de distribution des fréquences de la loi normale ( $n=$ 80; $G M Q_{0-20 j}=146 \mathrm{~g} / \mathrm{j} ; s d=77 \mathrm{~g} / \mathrm{j}$ ). le respect d'un effectif semblable (pour les 2 lots) dans chaque classe de $50 \mathrm{~g}$ de part et d'autre de la moyenne, et en se rapprochant au mieux de la loi normale. À l'intérieur de chaque classe, les individus ont été choisis par tirage aléatoire. Pour chacun des lots, 80 agneaux ont été retenus.

\section{Aliments}

Deux régimes alimentaires ont été distribués successivement. Le premier était un aliment commercial d'adaptation conçu pour assurer, après sevrage, la transition des agneaux vers une alimentation solide. Celui-ci était complémenté avec un prémix antibiosupplémenté afin de mieux maîtriser le risque sanitaire de groupe et les effets de stress. Il a été offert à raison de $12 \mathrm{~kg}$ en moyenne par agneau, couvrant la période des 22 premiers j d'engraissement. Le second régime, dit "d'engraissement" a été substitué progressivement au premier durant $5 \mathrm{j}$. La formulation de ce dernier aliment était différente pour chacun des lots : ALT, aliment commercial, a servi de témoin, ALS a été fabriqué en incorporant $3 \%$ de saindoux $(30 \mathrm{~g} / \mathrm{kg}$ d'aliment brut) sur une base isoénergétique et iso-azotée par rapport à l'aliment témoin. La composition chimique et la valeur nutritive des aliments font ressortir un taux de matières grasses supérieur pour ALS (5,5 vs 3,2\%), compensé en partie sur la base du poids par un taux de cellulose légèrement supérieur (tableau 1). La valeur énergétique est proche de 1 UFVI $\mathrm{kg}$ de MS et la teneur en MAT est de $16 \%$. Les acides gras des lipides alimentaires reflètent bien les caractéristiques du saindoux pour ce qui est de la teneur en acide palmitique. Par contre, les teneurs en acides stéarique et oléique sont faibles et celle d'acide linoléique élevée. En effet, le saindoux incorporé ne représente que $60 \%$ des lipides totaux dans ALS et les régimes diffèrent plus par leurs quantités respectives d'AG que par leur composition. La composition en acides gras de ALT est liée en partie à la présence de $1,1 \%$ de graisses d'os hydrogénées (bovosol) dans sa formulation. Les autres matières premières dans les 2 aliments ont la même origine mais leur proportion est différente pour permettre d'assurer des niveaux énergétique et azoté identiques. Au total, ALS contient $1,9 \%$ d'acides gras saturés et $3,6 \%$ d'acides gras insaturés contre 1 et $2,2 \%$ respec- 
Tableau I. Composition chimique et valeur nutritive des aliments expérimentaux. tr : trace, inférieure à $0,01 \%$.

\begin{tabular}{|c|c|c|c|}
\hline & \multirow[t]{2}{*}{$\begin{array}{c}\text { Aliment } \\
\text { de démarrage }\end{array}$} & \multicolumn{2}{|c|}{$\begin{array}{l}\text { Aliments de } \\
\text { "croissance-engraissement" }\end{array}$} \\
\hline & & $A L T$ (témoin) & ALS (saindoux) \\
\hline Mat sèche ( $\mathrm{g} / \mathrm{kg}$ aliment) & 902 & 905 & 909 \\
\hline \multicolumn{4}{|c|}{ Composition de la matière sèche $(\mathrm{g} / \mathrm{kg})$} \\
\hline Mat minérale & 90 & 82 & 80 \\
\hline Mat cellulosique & 67 & 79 & 92 \\
\hline Lignine & 29 & 30 & 34 \\
\hline Mat azotées $(\mathrm{N} \times 6,25)$ & 176 & 165 & 161 \\
\hline Mat grasse & 28 & 32 & 55 \\
\hline \multicolumn{4}{|l|}{ Valeur énergétique } \\
\hline UFV $/ \mathrm{kg}$ mat sèche & 0,96 & 0,97 & 0,98 \\
\hline EM en Mcal/kg mat sèche & 2,72 & 2,75 & 2,80 \\
\hline \multicolumn{4}{|c|}{ Composition en acides gras des matières grasses (en \% du poids des esters méthyliques totaux) } \\
\hline $\mathrm{C} 12$ & $\operatorname{tr}$ & 0,4 & 0,6 \\
\hline C14 & 1,2 & 1,8 & 1,4 \\
\hline $\mathrm{C}_{14}=1$ & 0,3 & $\operatorname{tr}$ & $\operatorname{tr}$ \\
\hline C15 & 0,4 & 0,3 & $\operatorname{tr}$ \\
\hline C16 & 20,0 & 21,1 & 22,4 \\
\hline $\mathrm{C} 16=1$ & 1,6 & 1,7 & 1,7 \\
\hline antéiso $\mathrm{C} 17$ & 0,5 & 0,4 & 0,2 \\
\hline $\mathrm{C} 17$ & 1,1 & 0,8 & 0,4 \\
\hline $\mathrm{C} 17=1$ & 1,1 & 0,6 & 0,4 \\
\hline C18 & 5,6 & 7,3 & 9,8 \\
\hline $\mathrm{C} 18=1$ & 27,8 & 30,1 & 30,1 \\
\hline $\mathrm{C} 18=2$ & 35,2 & 31,4 & 29,6 \\
\hline $\mathrm{C} 18=3$ & 4,9 & 3,7 & 3,2 \\
\hline $\mathrm{C} 20=1$ & 0,3 & 0,2 & 0,3 \\
\hline$\sum A G$ saturés & 28,8 & 32,1 & 34,7 \\
\hline$\sum \mathrm{AG}$ mono insaturés & 31,1 & 32,7 & 32,5 \\
\hline$\Sigma$ AG poly insaturés & 40,1 & 35,1 & 32,8 \\
\hline
\end{tabular}

tivement pour l'aliment témoin. L'acide palmitique est particulièrement bien représenté $(>20 \%$ des $A G$ totaux) et est 1,8 fois plus abondant dans l'aliment saindoux.

L'aliment d'engraissement, sous forme de granulés, a été distribué chaque jour, de façon à assurer en permanence une alimentation à volonté. Complémentairement, les agneaux ont eu accès à de la paille mise à leur libre disposition.

\section{Paramètres zootechniques}

Les paramètres de croissance mesurés individuellement sont : l'âge et le poids en début d'engraissernent (AGen, $P_{0}$ ), les poids à 20, 40, 60 j et à l'abattage $\left(P_{\mathrm{Aba}}\right)$, la durée d'engraissement (Duen), les vitesses de croissance dérivées (respectivement GMQ0-20, GMQ20-40, GMQ40-60, GMQ60-Aba, GMQ20-Aba, GMQ0$A b a)$, et la modalité de naissance (Nais). 
Les paramètres de carcasse ont été déterminés de la façon suivante:

- pour les aspects technologiques les contrôles ont été réalisés en abattoir, $18 \mathrm{~h}$ après ressuyage en chambre froide $\left(4^{\circ} \mathrm{C}\right)$. Ils ont porté sur le poids carcasse $\left(P_{\text {car }}\right)$, l'état d'engraissement (NGext), la notation de la conformation (Nconf) selon la classification Europa et le rendement commercial (Rdt);

- des mesures complèmentaires de l'adiposité ont permis d'apprécier l'épaisseur (en $\mathrm{mm}$ ) du gras dorsal (EGdo) à partir d'une fente au scalpel à hauteur des quatrième et cinquième vertèbres lombaires, l'importance des gras de carcasse de type périrénal (NGro, exprimé en g) et intercostal (NGic, exprimé selon une échelle de 1 à 5), la fermeté du gras externe (Nten, jugée d'après d'une échelle de 4 degrés : $1=$ gras mou, huileux; 4 = gras ferme, "caillé" (BouillierOudot et al, 1992)) et la couleur du gras de couverture (Ncoul, appréciée par référence au nuancier Munsell en 7 classes précisées par Bozzolo et al (1990): 1 = blanc; $7=$ bistre avec reflets verdâtres). Un seul et même examinateur expérimenté a procédé à l'ensemble des relevés sur carcasse.

L'efficacité alimentaire a été mesurée globalement par sommation des quantités d'aliment distribuées chaque jour et en la rapportant au nombre d'agneaux présents dans le lot. Les indices moyens de consommation $\left(/ c_{m}\right)$ ont été calculés par référence aux séquences de croissance $(0-20$ j, 20-40 j, 40-60 j, 60-Aba, 20Aba, 0-Aba) sur l'ensemble de l'échantillon initial $(n=2 \times 115)$. L'estimation d'un écart type $\left(S d\left(l_{c m}\right)\right)$ a été approchée par le calcul en considérant la relation des quantités ingérées (Q) avec le poids vif $(P)$ selon la fonction allométrique : $Q=a P^{b}$. Dans ces conditions $\mathrm{lcm}$ (moyen journalier) calculé entre $P_{i}$ et $P_{i+1}$ est égale à :

$$
\mathrm{d} Q / \mathrm{d} P=a b P_{m}^{b-1}
$$

En conséquence : $S d(l c)=l c_{m} \times(b-1) \times$ $S d\left(P_{m}\right) / P_{m}$. En effet, le développement limité de Taylor au voisinage de la moyenne donne, pour la fonction : $l c=\mathrm{t}(P)$, l'approximation:

lc $=l c_{m}+\left(P-P_{m}\right) \times \mathbf{f}^{\prime}\left(P_{m}\right)$, d'où :

$S d(I c) \approx S d(P) \times\left|f^{\prime}\left(P_{m}\right)\right|$

La valeur de $b$ est estimée entre $P_{0}$ et $P_{\text {Aba }}$ par l'ajustement des moindres carrés à partir de la forme linéaire de [1]:

$$
\operatorname{Ln}\left(/ c_{m}\right)=\operatorname{Ln}(a b)+(b-1) \times \operatorname{Ln}\left(P_{m}\right)
$$

Enfin : $S d\left(P_{m}\right)$

$=1 / 2 \sqrt{S d^{2}\left(P_{j}\right)+S d^{2}\left(P_{\dot{i}+1}\right)+2 r \times S d\left(P_{i}\right) \times S d\left(P_{i+1}\right)}$

avec $r$ : coefficient de corrélation entre $P_{i}$ et $P_{i+1}$.

Au total, l'écart type estimé $(S d)$ est égal à :

$S d\left(I c_{m}\right)=I c_{m}(b-1)$

$x \sqrt{S d^{2}\left(P_{i}\right)+S d^{2}\left(P_{i+1}\right)+2 r \times S d\left(P_{i}\right) \times S d\left(P_{i+1}\right)}$ $/\left(P_{j}+P_{j+1}\right)$

\section{Paramètres fermentaires}

Pour suivre l'évolution des profils fermentaires dans le rumen, plusieurs prélèvements de liquide ruminal ont été effectués à l'aide d'une sonde œsophagienne sur 6 agneaux dans chacun des lots $T$ et $S$, respectivement à 20,50 j et à l'abattage. Pour déterminer les concentrations molaires en acides gras volatils (AGV : acétique, propionique, isobutyrique, butyrique, isovalérique, valérique et caproïque) les prélèvements $(30 \mathrm{ml})$ ont été traités, conservés puis analysés par chromatographie en phase gazeuse, selon la méthode décrite par Jouany (1982).

Parallèlement, afin de suivre plus précisément l'évolution des faciès fermentaires durant la période de transition en fonction des régimes alimentaires distribués, 2 fermenteurs semicontinus (Rusitec) par régime ont été innoculés avec du jus de rumen prélevé durant la fin de la période de transition de post-sevrage (20 j). Le rythme d'introducion des sachets de nylon, respectivement remplis avec les aliments concernés (aliment de démarrage, puis ALT et ALS), complémentés avec $12 \%$ de paille fractionnée en brins d'environ $1 \mathrm{~cm}$, a été de $24 \mathrm{~h}$ pour un temps d'incubation de $48 \mathrm{~h}$, selon un protocole décrit par Blanchart et al (1989). L'observation a porté sur une période de 11 j pour l'aliment de "démarrage» et de $8 \mathrm{j}$ pour les aliments de "croissance-engraissement". Chaque jour, un prélèvement a été réalisé sur les effluents liquides stabilisés par adjonction de $\mathrm{HgCl}_{2}$ et les échantillons ont été stockés à $-18^{\circ} \mathrm{C}$ pour analyse ultérieure des AGV.

\section{Traitement des données}

Les observations de nature qualitative ou semiquantitative (Nten, Ncoul, Nais) sont comparées 
par l'examen de leur profil de répartition à l'aide du test du $\chi^{2}$. Dans le cas d'une répartition significativement différente, chaque pourcentage est comparé selon l'écart normal centré réduit.

Pour les paramètres quantitatifs, les comparaisons sont réalisées à l'aide du test $t$ de Snedecor (Snedecor et Cochran, 1957). Compte tenu de l'auto-corrélation des performances de croissance en fonction du temps d'engraissement, et de la non-sphéricité de la matrice de variance et covariance, les profils de croissance sont examinés au travers de l'ajustement polynomial individuel des $G M Q$ en fonction du temps et la comparaison des coefficients correspondants (Allen et al, 1983). De même, à partir de ces ajustements, sont calculés le GMQ maximum (GMQmax) et la durée d'engraissement associée (Jmax).

Les indices de consommation (lc) sont comparés, par période, à partir de l'estimation calculée de leur écart type.

Les paramètres fermentaires sont traités selon un plan factoriel 2(Régimes) $\times 3$ (Périodes) avec interaction et 6 répétitions, sur les données préalablement modifiées par la transformation angulaire $\arcsin \sqrt{x}$. Les profils de fermentation obtenus avec Rusitec, très homogènes selon les 2 répétitions, sont simplement comparés en fonction de l'évolution de leur moyenne en début de transition alimentaire.

\section{RÉSULTATS}

\section{Paramètres de croissance}

Les caractéristiques initiales d'élevage ( $P_{0}$, AGen, GMQ0-20) sont identiques pour les 2 lots (tableau II). Cependant, les jumeaux sont plus nombreux dans le lot témoin (70 vs $56 \%$ ), mais la répartition sur l'ensemble des naissances n'est pas significativement différente. En fait, ce critère intervient peu sur les paramètres de croissance et de carcasse $(r<10,16 \mid$ pour les 2 lots).

Les différentes séquences de croissance ne diffèrent pas significativement à $P<0,05$, exceptée la vitesse de croissance entre $60 \mathrm{j}$ et l'abattage supérieure de $7,6 \%$ pour le lot témoin $(P<0,02)$. Pour l'ensemble de la durée d'élevage en station ( $0 \mathrm{j}-\mathrm{Aba})$ et sur la période propre à la consommation des régimes testés $(20 \mathrm{j}-$ Aba), les vitesses de croissance sont semblables et atteignent en moyenne 310 et $361 \mathrm{~g}$ respectivement. La durée d'engraissement moyenne est en conséquence identique (92 j; $c v=14 \%$ ).

L'observation des coefficients moyens de l'ajustement polynomial selon les lots (tableau III) ne permet pas de déceler une évolution très différente des courbes de croissance (fig 2). Une tendance apparaît cependant en phase terminale par le biais des 2 coefficients des termes quadratique et cubique $(P<0,15 ; P<0,09)$ : elle traduit une forme plus régulière obtenue avec le lot complémenté au saindoux (fig 2), alors que pour le régime témoin, la croissance serait biphasique.

\section{Paramètres de carcasse}

Les différents critères technologiques (Pcar, Rdt, Nconf) et d'engraissement ne diffèrent pas d'un lot à l'autre (tableau IV).

En ce qui concerne la qualité des gras externes (tableau $V$ ), la répartition des profils de tenue selon les lots extériorise un effet globalement significatif du saindoux $(P<0,02)$. Celui-ci permet d'obtenir une plus grande proportion de carcasses fermes (NTen3 et NTen4) : 61,3 contre $46,3 \%$ pour le lot témoin $(P<0,06)$ et surtout moins de carcasses classées Nten 2 (27,5 contre $48,7 \% ; P<0,01)$.

II en va de même pour les caractéristiques de coloration du tissu gras externe $(P<0,03$; tableau $V)$. Le régime enrichi en saindoux produit plus de carcasses blanches (Ncoul1 : 19 vs $6 \% ; P<0,02$ ), ceci essentiellement en substitution de la teinte bistre claire (Ncou/4 : 15 vs 30\%; $P<0,03)$. Les autres nuances ne présen- 
Tableau II. Incidence des régimes alimentaires sur les paramètres de croissance. $S^{\star}$ significatif à $P<$ $0,05\left(^{*}\right)$; ns : non significant à $P<0,20$ (test de Student).

Caractéristiques initiales d'élevage des 2 lots mis en comparaison

\begin{tabular}{|c|c|c|c|c|c|c|}
\hline \multirow{3}{*}{\multicolumn{2}{|c|}{$\begin{array}{l}\text { Effectif } \\
\text { Poids en début d'engraissement (kg) } \\
\text { Age en début d'engraissement }(\mathrm{j})\end{array}$}} & 80 & & 80 & & \\
\hline & & 12,2 & $(0,20)$ & 12,2 & $(0,17)$ & ns \\
\hline & & 28 & $(0,36)$ & 28 & $(0.37)$ & ns \\
\hline \multirow[t]{3}{*}{ Mode de naissance (fréquence) : } & 1 & $25 \%$ & & $36 \%$ & & $i^{\text {ns }}$ \\
\hline & 2 & $70 \%$ & & $56 \%$ & & Zns \\
\hline & 3 & $4 \%$ & & $8 \%$ & & is \\
\hline Gain moyen quotidien $(\mathrm{g})(0-20 \mathrm{j})$ & & 145 & $(8,7)$ & 147 & $(8,5)$ & ns \\
\hline Indice de consommation $(0-20 \mathrm{j})$ & & 3,18 & $(0,24)$ & 2,85 & $(0,26)$ & ns \\
\hline
\end{tabular}

Paramètres d'élevage durant la période d'engraissement comparée

Gain moyen quotidien (GMQ en $\mathrm{g} / \mathrm{j}$ )

\begin{tabular}{|c|c|c|c|c|c|}
\hline$G M Q_{20-40}$ & 356 & $(8,9)$ & 354 & $(8,2)$ & ns \\
\hline$G M Q_{40-60 j}$ & 364 & $(11,8)$ & 380 & $(9,6)$ & ns \\
\hline$G M Q_{60 j-A b a}$ & 370 & $(8,7)$ & 344 & $(7,0)$ & * \\
\hline$G M Q_{0 j-A b a}$ & 313 & $(4,3)$ & 308 & $(4,0)$ & ns \\
\hline$G M Q_{20 j-A b a}$ & 366 & $(5,6)$ & 356 & $(5,1)$ & ns \\
\hline Poids abattage $(\mathrm{kg})$ & 40,5 & $(0,1)$ & 40,4 & $(0,1)$ & ns \\
\hline Durée d'engraissement $(j)$ & 92 & $(1,5)$ & 93 & $(1,5)$ & ns \\
\hline \multicolumn{6}{|c|}{ Indice de consommation $(/ c=\delta \mathrm{MSi} / \delta P d s)$} \\
\hline Ic $20-40 \mathrm{j}$ & 2,54 & $(0,22)$ & 2,81 & $(0,26)$ & ns \\
\hline Ic $40-60 \mathrm{j}$ & 3,79 & $(0,34)$ & 3,35 & $(0,28)$ & ns \\
\hline Ic $60 \mathrm{j}-\mathrm{Aba}$ & 3,56 & $(0,15)$ & 4,14 & $(0,15)$ & ns \\
\hline Ic $0 \mathrm{j}$-Aba & 3,28 & $(0,07)$ & 3,47 & $(0,08)$ & $9 \%$ \\
\hline Ic $20 \mathrm{j}-\mathrm{Aba}$ & 3,35 & $(0,09)$ & 3,59 & $(0,10)$ & $8 \%$ \\
\hline$b$ (coef d'allométrie $20 \mathrm{j}$-Aba) & 1,52 & $(0,24)$ & 1,60 & $(0,04)$ & ns \\
\hline
\end{tabular}

Tableau III. Profils de croissance - comparaison des coefficients de l'ajustement polynomial des GMQ individuels en fonction de la durée d'engraissement et selon la nature du régime alimentaire $\left(y=a_{0}+\right.$ $\left.a_{1} t+a_{2} f^{*}+a_{3} \beta\right) . S^{\star}(\%)$ seuil de signification; ns : non significatif à $P<0,20$ (test de Student).

\begin{tabular}{lcllll}
\hline Régimes & $\begin{array}{c}\text { Témoin (es) } \\
(\mathrm{n}=80)\end{array}$ & $\begin{array}{c}\text { Saindoux }(e s) \\
(\mathrm{n}=80)\end{array}$ & $S^{*}(\%)$ \\
\hline Coefficients de l'ajustement & & & & & \\
$a_{0}$ & -95 & $(24)$ & -54 & $(32)$ & $\mathrm{ns}$ \\
$a_{1}$ & 29,7 & $(3,0)$ & 24,0 & $(3,6)$ & $\mathrm{ns}$ \\
$a_{2}$ & $-0,61$ & $(0,10)$ & $-0,41$ & $(0,09)$ & $15 \%$ \\
$a_{3}$ & 0,004 & $(0,0007)$ & 0,002 & $(0,0008)$ & $9 \%$ \\
Gain moyen quotidien maximum (GMOmax) (g/i) & 427 & $(8)$ & 424 & $(8)$ & $\mathrm{ns}$ \\
Durée d'engraissement jusqu'au GMOmax (j) & 43 & $(1,6)$ & 45 & $(1,5)$ & $\mathrm{ns}$ \\
\hline
\end{tabular}




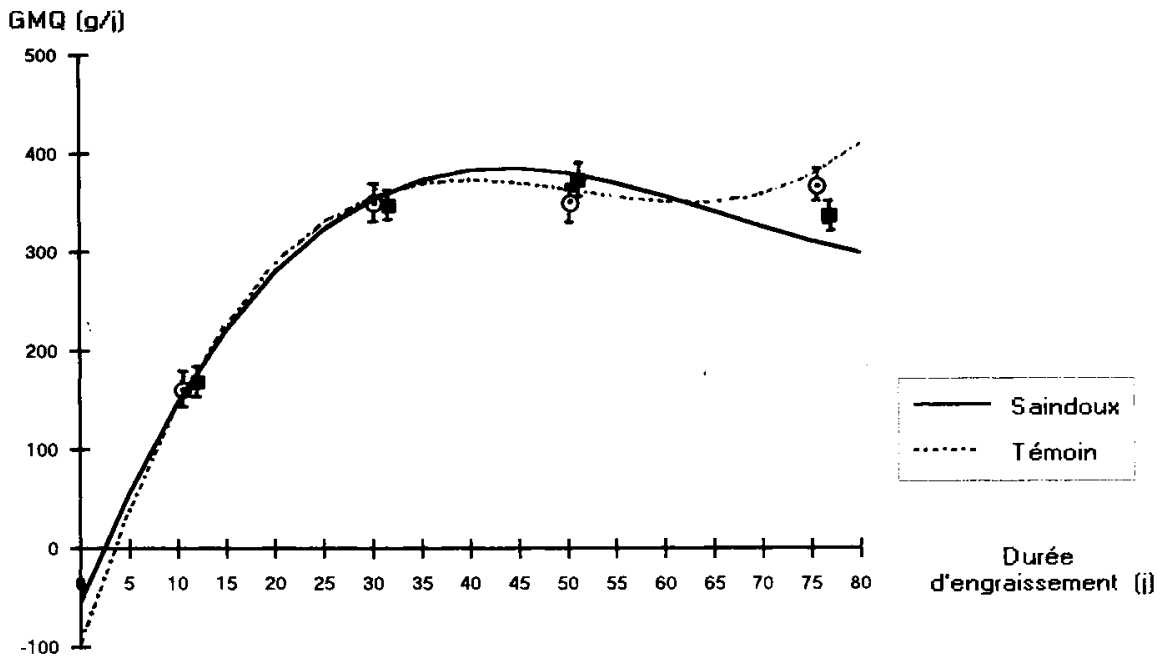

Fig 2. Profil de croissance des agneaux engraissés avec les régimes "témoin" et "saindoux" en fonction de la durée d'engraissement. Ajustement polynomial : $y=a_{0}+a_{1} t+a_{2} t^{2}+a_{3} t^{3}$. O, $\mathbf{v}: G M Q$ moyen observé $\pm 2 \times$ (es) dans les lots témoin et saindoux respectivement.

Tableau IV. Incidence des régimes alimentaires sur les paramètres de carcasse. " $S(\%)$ seuil de signification; ns (non significatif à $P<0,20$ ) (test de Student).

\begin{tabular}{lccc}
\hline Régimes & $\begin{array}{c}\text { Témoin (es) } \\
(\mathrm{n}=80)\end{array}$ & $\begin{array}{c}\text { Saindoux (es) } \\
(\mathrm{n}=80)\end{array}$ & $S^{*}(\%)$ \\
\hline
\end{tabular}

\section{Paramètres}

\begin{tabular}{lccccc} 
Poids carcasse $(\mathrm{kg})$ & 19,9 & $(0,1)$ & 19,9 & $(0,1)$ & $\mathrm{ns}$ \\
Rendement carcasse $(\%)$ & 52 & $(0,2)$ & 52 & $(0,2)$ & $\mathrm{ns}$ \\
Note de conformation & 2,4 & $(0,09)$ & 2,3 & $(0,08)$ & $\mathrm{ns}$ \\
Note de gras externe & 2,4 & $(0,07)$ & 2,5 & $(0,07)$ & $\mathrm{ns}$ \\
Épaisseur de gras dorsal $(\mathrm{mm})$ & 2,3 & $(0,17)$ & 2,6 & $(0,2)$ & $\mathrm{ns}$ \\
Note de gras périrenal $(\mathrm{g})$ & 222 & $(14)$ & 230 & $(14)$ & $\mathrm{ns}$ \\
Note de gras intercostal & 2,9 & $(0,07)$ & 2,8 & $(0,08)$ & $\mathrm{ns}$ \\
\hline
\end{tabular}

tent pas de proportions significativement différentes.

\section{Paramètres fermentaires dans le rumen}

Les évolutions en fonction du temps sont assez comparables d'un régime à l'autre, avec un enrichisssement de la proportion d'acide acétique et une dimi- nution de celle d'acide propionique (approchant respectivement 52 et $35 \%$ en fin d'engraissement; tableau VI). L'interaction fait ressortir une diminution de la teneur de l'acide propionique plus lente dans le temps avec le régime témoin, soit après $30 \mathrm{j}$ de consommation (42,7 vs $36,2 \% ; P<0,05)$. Pour les acides butyrique et valérique la tendance est inversée avec, respectivement après $30 \mathrm{j}$ : 
Tableau V. Incidence du régime d'engraissement sur la qualité du tissu gras sous-cutané de carcasses d'agneaux (tenue, couleur) - Profils de répartition en $\%(n=2 \times 80)$. a,b Pourcentage significativement différent à $P<0,05$.

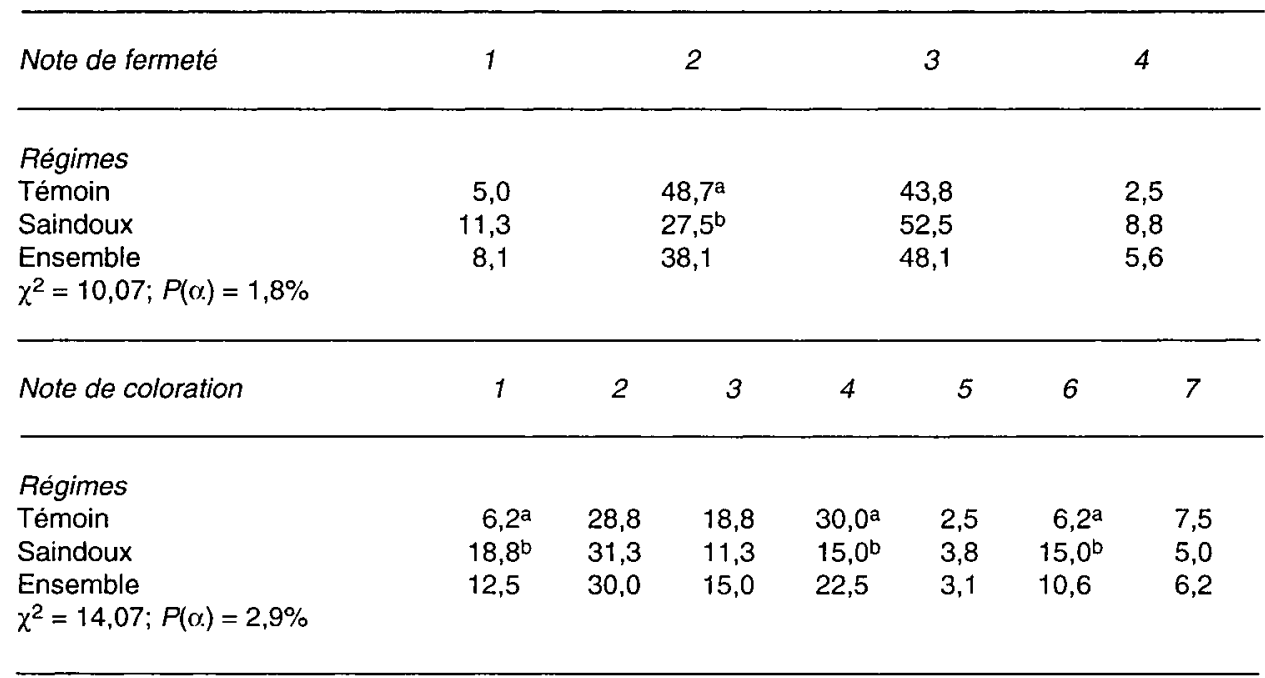

Tableau VI. Profils de répartition des AGV fermentaires (\% molaires) selon le régime alimentaire et selon la durée d'engraissement $(n=6)$. $F$ : signification du $F$ de Snedecor au seuil de $P<0,001\left(^{* * *}\right)$, $P<0,01\left(^{* *}\right), P<0,05\left(^{*}\right)$, ns (non significatif) pour les effets : Périodes (Pér), Interaction (Inter), Régimes, calculés à partir des valeurs transformées $\arcsin \sqrt{x}$. a. b différences significatives au seuil $P=$ 0,05 (Newmans-Keuls).

\begin{tabular}{|c|c|c|c|c|c|c|c|c|c|}
\hline \multirow[b]{2}{*}{$\begin{array}{l}\text { Durée } \\
\text { d'engraissement }\end{array}$} & \multirow{2}{*}{$\frac{\text { Transition }}{20 \mathrm{j}}$} & \multicolumn{2}{|c|}{$\begin{array}{l}\text { Régimes } \\
\text { Témoin }\end{array}$} & \multicolumn{3}{|c|}{ Saindoux } & \multicolumn{3}{|c|}{$F$} \\
\hline & & $50 j$ & Abattage & $50 \mathrm{j}$ & Abattage & es & Pér & Inter & Régimes \\
\hline \multicolumn{10}{|l|}{ AGV (\% molaires) } \\
\hline Acétique & 39,9 & 46,1 & 51,0 & 46,9 & 52,2 & 0,6 & $\star \star \star \star$ & ns & ns \\
\hline Propionique & $44,4^{\mathrm{a}}$ & $42,7^{a}$ & $34,2^{\mathrm{b}}$ & $36,2^{b}$ & $35,1^{\mathrm{b}}$ & 0,7 & $\star \star \star *$ & * & ns \\
\hline Isobutyrique & 0,2 & 1,3 & 1,2 & 0,2 & 1,1 & 0,8 & $* * *$ & $\mathrm{~ns}$ & ns \\
\hline Butyrique & $11,1^{\mathrm{a}}$ & $6,7 \mathrm{~b}$ & $8,0^{\mathrm{ab}}$ & $10,5^{\mathrm{a}}$ & $6,4^{b}$ & 0,8 & $\star \star \star *$ & * & ns \\
\hline Isovalérique & 0,3 & 0,2 & 1,8 & 0,3 & 1,4 & 0,5 & $* * *$ & ns & ns \\
\hline Valérique & $3,7^{a b}$ & $2,6^{a}$ & $3,4^{a b}$ & $5,3^{b}$ & $3,4^{a b}$ & 0,8 & ns & * & * \\
\hline Caproïque & 0,4 & 0,4 & 0,3 & 0,6 & 0,3 & 0,8 & * & ns & ns \\
\hline
\end{tabular}

6,7 et $2,6 \%$ vs 10,5 et $5,3 \%$ pour les lots témoin et saindoux respectivement $(P<0,05)$.
Dans les fermenteurs de type Rusitec, le taux de propionate diminue tandis que ceux d'acides acétique et caproïque aug- 
mentent, notamment pour le régime ALS (fig 3). En revanche les teneurs des acides en $\mathrm{C} 4$ et $\mathrm{C} 5$ restent stables. Globalement, il apparaît que les profils ne diffèrent pas d'un régime à l'autre. Par ailleurs, les proportions sont différentes de celles observées in vivo après une phase de stabilisation des fermenteurs correspondant à leurs caractéristiques propres.
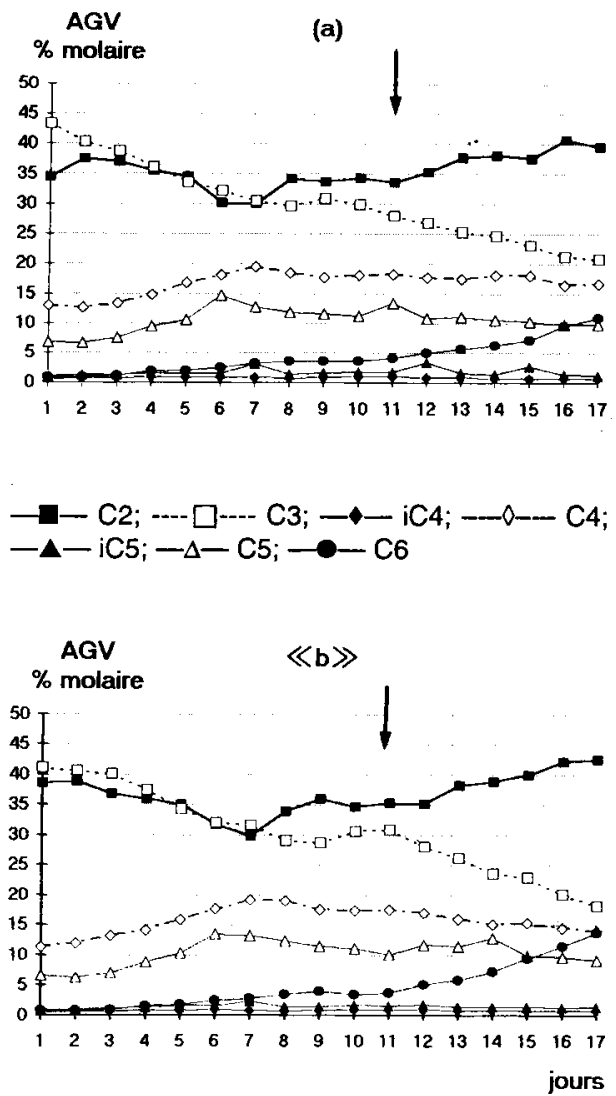

Fig 3. Évolution des profils fermentaires (\% AGV) dans les incubateurs semi-continus (Rusitec) en fonction de la nature des aliments, au moment de la transition entre aliments de démarrage et d'“engraissement-finition". a) Régime d'engraissement témoin (ALT); b) régime d'engraissement pourvu en saindoux (3\%) (ALS). $\downarrow$ : Passage de l'aliment de "démarrage" aux aliments d'cengraissement" (ALT et ALS).

\section{Indices de consommation}

Si l'on considère les 3 séquences de croissance de la période d'engraissement, il n'apparaît de différence significative que pour l'indice de consommation $/ c_{60-A b a}$ entre les 2 lots. Cet indice est détérioré dans le lot saindoux $(+0,61$ point $)$, précédé cependant par une évolution inverse $(-0,4$ point). Par contre, une brusque élévation est marquée au cours de la période 40-60 j dans les 2 lots $(3,6$ vs 2,7), c'est-à-dire à un poids vif moyen correspondant dans les 2 cas à $26 \mathrm{~kg}$.

En conséquence, $l_{0 \text {-Aba }}$ et $l_{c_{20} \text {-Aba }}$ sont identiques pour les 2 lots et atteignent respectivement 3,37 et $3,47 \mathrm{~kg}$ de MS ingérée/kg de gain de poids vif. De même, les coefficients d'allométrie correspondant à la période d'engraissement, après transition (entre 48 et $120 \mathrm{j}$ de vie) sont semblables ( $b=1,52$ et $b=1,60$ pour les lots ALT et ALS respectivement).

\section{DISCUSSION}

\section{Croissance des animaux}

L'incorporation de saindoux à raison de $3 \%$ de la matière brute de l'aliment, soit $60 \%$ des lipides totaux $(5,5 \%$ de la MS) dans le régime de croissance-finition d'agneaux de bergerie de type Lacaune et sevrés précocement, n'induit pas de modification significative $(P<0,05)$ des performances de croissance, même lorsque les agneaux sont soumis à un rythme intensif (361 $\mathrm{g} / \mathrm{j}$ en moyenne durant cette phase) en comparaison d'un régime commercial contenant $3 \%$ de matières grasses. Ponctuellement, le meilleur GMQ des agneaux du lot témoin en phase terminale d'engraissement pourrait correspondre à un effet de croissance compensatrice si on se 
réfère à l'évolution des courbes de croissance avec le temps. Celle-ci permet aux agneaux de rattraper le retard de la période précédente. La mise en évidence de cette différence significative est aussi à relier à la moindre variabilité intralot en période de finition. Ces régimes permettent de produire des agneaux de boucherie de $20 \mathrm{~kg}$ de poids-carcasse en $93 \mathrm{j}$ d'engraissement, soit à un âge moyen de $120 \mathrm{j}$. Par ailleurs, les caractéristiques quantitatives de l'adiposité des carcasses ne sont pas affectées et ne nuisent pas à leurs qualités commerciales (NGext $<2,5$ ). De plus, les indices de consommation enregistrés sont conformes à ceux obtenus par ailleurs pour cette même catégorie d'agneaux (ITCF, 1981) et ne diffèrent pas entre ALT et ALS sur l'ensemble de l'engraissement. Cependant, la reprise de croissance comparative du lot témoin en période de finition caractérisée par un indice de consommation moindre $\left(/ c_{0-A b a}=3,56\right.$ vs 4,14$)$, pourrait être liée à un mécanisme d'interrelation hormono-nutritionnelle. En effet, chez les mâles, l'état prépubertaire et le niveau élevé de propionate sérique pourraient exacerber l'activité de l'hormone de croissance dans le cas d'une lipémie plus faible (Waghorn et al, 1987).

En conséquence, un taux d'incorporation supérieur (5-7\%) de saindoux dans l'aliment pourrait être envisagé, d'autant que pour un régime de finition, une éventuelle diminution de la digestibilité des constituants pariétaux (Palmquist et Conrad, 1980; McAllan et al, 1983; Sutton et al, 1983; Bock et al, 1991), ne constitue pas un problème majeur. De même, Jenkins et Jenny (1989) montrent que, chez la vache laitière, l'incorporation de graisses préalablement hydrogénées dans l'aliment à des taux de 3 et $5 \%$, supprime les effets de réduction de l'ingestion habituellement constatée avec l'emploi des corps gras de type insaturés.

\section{Qualité du gras de couverture}

Pour cette catégorie d'agneaux ayant une forte prédisposition à produire des carcasses de médiocre qualité sur le plan de la tenue $(54 \%$ dans le lot témoin, ce qui rejoint les observations de De Boisseson et Mahmoudi (résultats non publiés)), l'addition de saindoux améliore sensiblement la proportion des carcasses de bonne valeur commerciale (Nten3 et Nten4 : $+15 \%$ ). De même, l'éventail des colorations observées par référence au nuancier Munsell, montre la faible proportion de carcasses claires dans le lot témoin $(35 \%)$, catégorie recherchée par la profession, confirmant un précédent constat réalisé sur des agneaux de Roquefort (Bozzolo et al, 1991). L'introduction de saindoux permet d'accroître ce taux à $50 \%$. Cette amélioration des qualités du gras externe rejoint l'observation de Solomon et Lynch (1988).

La mise en évidence d'une réduction importante de la proportion de carcasses bistre clair ( $N$ coul4, réf 7.5YR8/6: $-15 \%$ ), associée à une plus grande fréquence de gras fermes, laisse supposer qu'il s'agit d'une diminution de la part des triglycérides à faible point de fusion, riches en acides gras insaturés et/ou ramifiés (l'Estrange et Mulvihill, 1975; Prache et al, 1990; Bouillier-Oudot et al, 1992). Bock et al (1991) constatent d'ailleurs un accroissement de la proportion d'acide stéarique et une diminution de celle de l'acide palmitoléique dans le tissu gras intermusculaire. Cette orientation peut être expliquée par l'importance croissante de la part des acides gras alimentaires dans la constitution des dépôts adipeux en période de finition (Christie, 1981; Aurousseau, 1986). Non seulement l'aspect quantitatif de l'incorporation des matières grasses dans l'aliment est à souligner, mais aussi les caractéristiques propres du saindoux. Contrairement à la plupart des AG en C18, 
l'acide palmitique ne semble pas être remanié et conduit à des graisses de dépôt à haut point de fusion (Christie, 1981). Dans notre essai, l'apport quantitatif d'acides stéarique et palmitique dans l'aliment "saindoux" est 2 et 1,8 fois plus élevé que dans ALT $(0,54$ vs $0,24 \%$ et 1,23 vs $0,67 \%$ respectivement). Devier et Pfander (1974) signalaient déjà l'accroissement des proportions d'AG saturés dans le tissu gras, en particulier de $\mathrm{C} 18=0$, avec l'incorporation de suif dans l'aliment (2,5 et $4 \%$ ) en comparaison de celle d'huile de maïs.

\section{Paramètres fermentaires}

Le niveau des taux d'AGV dans le liquide du rumen et l'évolution de leur profil selon la durée d'engraissement ne fait pas ressortir de différence importante entre régimes alimentaires, rejoignant les résultats de Bock et al (1991) sur bouvillons, de Chalupa et al (1986) sur vache laitière et les résultats sur vache laitière et sur mouton rapportés par Bauchart et al (1985) pour des taux d'incorporation de matières grasses compris entre 2 et $10 \%$. Les profils évolutifs mis en évidence par les fermentations in vitro, durant la courte période de transition, confirment les tendances observées in vivo, bien que les taux d'AGV soient différents. Par contre, l'évolution des teneurs en acide caproïque n'est pas constatée in vivo après $30 \mathrm{j}$ d'engraissement; ce qui est certainement dû à une déviation propre aux fermenteurs. La présence d'avoparcine pourrait d'ailleurs contribuer à amplifier ce phénomène par un effet de majoration de son activité dans cette enceinte non conforme à la réalité du rumen (absorption transépithéliale, paramètres des flux alimentaires, caractéristiques de la salive).

Le taux élevé d'acide propionique $(>34 \%$ ) dans le contenu ruminal apparaît comme un facteur favorisant l'apparition de carcasses défectueuses si on se réfère aux recommandations d'Ørskov et al (1975) pour qui des taux supérieurs à 30\% sont critiques. Cependant, nous remarquons que, même dans ces conditions défavorables pour la qualité des dépôts gras, les acides gras alimentaires longs et saturés permettent de réduire la proportion des carcasses défectueuses. Les teneurs en acides butyrique et isobutyrique sont nettement moindres (pratiquement du simple au double) que celles obtenues par Murphy et L'Estrange (1977) avec des régimes céréaliers, renforçant en conséquence la présence relative des $A G V$ acétique et propionique. Dans ces conditions, l'utilisation métabolique de l'acide propionique par le foie pourrait être plus élevée, réduisant ainsi la synthèse d'acides gras ramifiés par le tissu adipeux, générateurs des graisses molles. En effet, Aiello et al (1989) et Demigné et al (1991), ont montré une action inhibitrice du butyrate sur la transformation hépatique du propionate en glucose.

Ce constat irait dans le sens d'un effet exercé par les acides gras longs sur l'augmentation des taux d'acides propionique et valérique au détriment de l'acide butyrique (Church, 1979). En fait, l'absence de différence prononcée entre nos 2 régimes ne permet pas d'aboutir à cette conclusion. $\mathrm{Ce}$ profil particulier constitue probablement un effet secondaire lié à la présence d'avoparcine dans les 2 aliments (0,2\% d'avotan $/ \mathrm{kg}$ d'aliment). Cet additif est connu pour son action sélective sur la flore en favorisant la production d'acide propionique au détriment de l'acide butyrique (Macgregor, 1983). Au contraire, la supplémentation des régimes en saindoux met à jour une légère tendance à l'abaissement plus rapide du taux d'acide propionique et à l'élévation des taux d'acides butyrique et valérique au cours de l'engraissement. La différence de qualité 
de carcasse observée entre lot témoin et lot supplémenté serait donc plutôt à rechercher dans la plus forte participation des AG longs saturés alimentaires (notamment, l'acide palmitique) à la construction des triglycérides adipocytaires que dans une éventuelle modification des orientations fermentaires dans le rumen.

\section{CONCLUSION}

Les nombreux essais de supplémentation en lipides alimentaires non protégés, réalisés antérieurement chez les ruminants et les agneaux en particulier font ressortir que les modifications sur la composition en acides gras des tissus adipeux sont mineures lorsque leurs taux d'incorporation $(<10 \%)$ sont compatibles avec une ingestibilité normale (Church, 1979; Christie, 1981; Bauchart et al, 1985). Ce constat est souvent expliqué d'une part, par l'hydrogénation des $A G$ insaturés dans le rumen et/ou leur incorporation préférentielle dans les esters de cholestérol des particules lipoprotéiques, fractions lipidiques non hydrolysées par la lipoprotéine lipase (Christie et al, 1986), ne contribuant pas ainsi à la synthèse des triglycérides neutres de réserve et, d'autre part, par les niveaux de digestibilité et les remaniements potentiels des AG saturés. Dans le cas d'agneaux engraissés intensivement, issus de races au développement tardif comme c'est le cas des mâles Lacaune, l'emploi de saindoux dans l'aliment d'engraissement, riche en $A G$ longs saturés (spécialement en C16 = 0), permet d'enregistrer un effet probant sur la fermeté et la coloration du tissu gras sous-cutané. L'observation de performances de croissance non modifiées, avec un profil d'évolution plus régulier en fonction de la durée d'engraissement et des indices de consommation non détériorés, conduit à penser qu'une augmentation du taux d'incor- poration du saindoux pourrait renforcer l'amélioration constatée sur l'apparence des gras externes de carcasse.

\section{REMERCIEMENT}

Travail subventionné par le Conseil régional de Midi-Pyrénées pour l'encouragement de la Recherche scientifique $\left(\mathrm{n}^{\circ}\right.$ de contrat 9000743 ).

\section{RÉFÉRENCES}

Allen OB, Burton JH, Holt JD (1983) Analysis of repeated measurements from animal experiments using a polynomial regression. $J$ Anim Sci 57, 765-770

Aiello RJ, Armentano LE, Bertics SJ, Murphy AT (1983) Volatile fatty acid uptake and propionate metabolism in ruminant hepatocytes. J Dairy Sci 72, 942-949

Andrews RP, Ørskov EE (1970) The nutrition of the early weaned lamb: II. The effect of dietary protein concentration, feeding level and sex on body composition at two live weights. J Agric Sci Camb 75, 19-26

Annison EF (1984) The metabolism of neutral acidic lipids by tissues of the ruminant. In: Herbivore nutrition in the subtropics and tropics (FMC Gilchrist, RI Mackie, eds) The Science Press, Craighall, South Africa, 549-570

Aurousseau B (1981) Élaboration des lipides corporels et valeur des carcasses des ruminants. Bull Tech CRVZ Theix INRA 45, 43-50

Aurousseau B (1986) Influence des facteurs d'élevage sur l'état d'engraissement et la qualité des carcasses chez les ovins. In : Qualités des produits chez les ovins et les caprins. $11^{\text {es }}$ Journ rech ovine et caprine, INRA-ITOVIC, SPEOC, Paris, 210-235

Aurousseau B, Thériez M, Daniel M (1973) Influence de la nature des matieres grasses incorporées dans l'aliment d'allaitement sur le métabolisme lipidique de l'agneau de bergerie. Ann Biol Anim Biochim Biophys 13, 93105

Aurousseau B, Vermorel $M$, Thériez $M$, Vezinhet A (1989) Effects of substitution of tricaprylin or coconut oil for tallow in milk replacers of- 
fered to preruminant lambs. Ann Zootech 38, 49-59

Bauchart G, Doreau M, Legay-Carmier F (1985) Utilisation digestive des lipides et conséquences de leur introduction sur la digestion du ruminant. Bull Tech CRVZ Theix INRA 61, 65-77

Blanchart $G$, Durand $M$, Barry $J L$, BouillierOudot M, Jouany JP (1989) Intérêts et limites des fermenteurs à flux semi-continu de type Rusitec dans l'étude des fermentations du rumen. Ann Zootech 38, 285-314

Bock BJ, Harmon DL, Brandt RT, Schneider Jr, Schneider JE (1991) Fat source and calcium level effects on finishing steer performance, digestion and metabolism. J Anim Sci 69, 2211-2224

Bouillier-Oudot M, Bozzolo G, Phrem G (1992) Optimisation du jugement de la fermeté du gras de couverture des carcasses d'agneaux de bergerie. Ann Zootech, 185-201

Bozzolo $G$, Bouillier-Oudot M, De Boisseson E, Ghassan M, Grasset D (1990) Influence des performances zootechniques sur les caractéristiques des tissus adipeux d'agneaux de bergerie, sevrés précocement et alimentés avec un régime à forte concentration énergétique. Ann Zootech 39, 77-94

Bozzolo G, Bouillier-Oudot M, Aoun M, Grasset $D$, Manse $H$ (1991) Incidence des acides gras alimentaires à chaîne moyenne, incorporés dans le régime de transition en postsevrage, sur les caractéristiques de croissance et de carcasse d'agneaux sevrés précocement et engraissés intensivement en bergerie en été. Ann Zootech 40, 85-105

Bremner HA, Ford AL, Mac Farlan JJ, Ratcliff D, Russel NT (1976) Meat with high linoleic acid content: oxidative changes during frozen storage. J Food Sci 41, 757-761

Busboom JR, Miller CJ, Field RA, Crouse JD, Riley ML, Nelms GE, Ferell CL. (1981) Characteristics of fat from heavy ram and wether lambs. J Anim Sci 52, 83-92

Cameron CW, Hogue DE (1968) Effect of varying dietary corn oil and hay grain ratio on lamb growth and fat characteristics. I Anim Sci 27, 553-556

Cera KR, Mahan DC, Reinhart CA (1989) Apparent fat digestibilities and performances responses of post-weaning swine fed diets sup- plemented with coconut oil, corn oil or tallow. $J$ Anim Sci 67, 2040-2047

Chalupa W, Vecchiarelli B, Elser AE, Kronfeld DS, Sklan D, Palmquist DL (1986) Ruminal fermentation in vivo as influenced by long chain fatty acids. J Dairy Sci 69, 1293-1301

Christie WW (1981) The effects of diet and other factors on the lipid composition of ruminant tissues and milk. In: Lipid Metabolism in ruminant animals (WW Christie, ed) Pergamon Press, Oxford, 193-225

Christie WW, Noble RC, Clegg RA (1986) The hydrolysis of very low density lipoproteins and chylomicrons of intestinal origin by lipoprotein lipase in ruminants. Lipids 21, 252-253

Church DC (1979) Lipid utilization and requirements. In: Digestive physiology and nutrition of ruminants: nutrition. Oxford Press, Portland, OR, vol 2, 167-186

Crouse JD, Field RA, Chant JL, Ferrell CL, Smith GM, Harrison VL (1978) Effect of dietary energy intake on carcass composition and palatability of different weight carcasses from ewe and ram lambs. J Anim Sci 47, 1207-1218

Demigné $C$, Yacoub $C$, Morand $C$, Rémésy $C$ (1991) Interaction between propionate and amino acid metabolism in isolated sheep hepatocytes. Br J Nutr 65, 301-317

Devendra C, Lewis D (1974) The interaction between dietary lipids and fiber in the sheep. Anim Prod 19, 67-76

Devier CV, Pfander WH (1974) Source and level of dietary fat on fatty acid and cholesterol in lambs. J Anim Sci 38, 669-675

Faustman C, Cassens RG, Schaefer DM, Buege DR, Williams SN, Scheller KK (1989) Improvement of pigment and lipid stability in Holstein steer beef by dietary supplementation with vitamin E. $J$ Food Sci 54, 858-862

Field RA, Williams JC, Ferell CL, Crouse JD, Kunsman JE (1978) Dietary alteration of palatability and fatty acids in meat from light and heavy weight ram lambs. J Anim Sci 47 , 858-864

Garton GA, Howell FDD, Duncan WRH (1972) Influence of dietary volatile fatty acids on the fatty acid composition of lamb triglycerides with special reference to the effect of propionate on the presence of branched chain components. Br J Nutr 28, 409-416 
ITCF (1981) Maïs grain, plante entière, épi pour l'engraissement des agneaux. In : Utilisation des céréales et des fourrages par les animaux. Rapport 1981 sept (8), 39

Jenkins TC, Palmquist DL (1982) Effect of added fat and calcium on in vitro formation of insoluble fatty acid soaps and cell wall digestibility. J Anim Sci 55, 957-963

Jenkins TC, Jenny BF (1989) Effect of hydrogenated fat on feed intake, nutrient digestion and lactation performance of dairy cows. J Dairy Sci 72, 2316-2324

Jouany JP (1982) Volatile fatty acid and alcohol determination in digestive contents silage juices, bacterial cultures and anaerobic fermentor contents. Sci Alim 2, 131-144

Kowalczyk JE, Orskov ER, Robinson JJ, Stewart CS (1977) Effect of fat supplementation on voluntary intake and rumen metabolism in sheep. Br J Nutr 37, 251-257

L'Estrange JL, Mulvihill TA (1975) A survey of fat characteristics of lambs with particular reference to the soft fat condition in intensively fed lambs. J Agric Sci (Camb) 84, 281-290

Mac Allan AB, Knight R, Sutton JD (1983) The effect of free and protected oils on the digestion of dietary carbohydrates between the mouth and duodenum of sheep. $\mathrm{Br} J$ Nutr 49, 433-440

Macgregor RC (1983) Growth promoters and their importance in ruminant livestock production. In: Recent advances in animal nutrition (W Haresign, ed) Butterworths, UK, 17, 163-177

Mahan DC (1991) Efficacy of initial postweaning diet and supplemental coconut oil or soybean oil for weanling swine. J Anim Sci 69 , 1397-1402

Miller CJ, Rice RW (1967) Lipid metabolism in lambs as affected by fattening rations of roughage and concentrate. J Anim Sci 26 , 1153-1159

Miller GJ, Kunsman JE, Field RA (1980) Characteristics of subcutaneous fat in ram lambs fed corn and corn-silage diets. J Food $\mathrm{Sci}$ 45, 279-282

Molénat G, Thériez M (1973) Influence du mode d'élevage sur la qualité de la carcasse de l'agneau de bergerie. Ann Zootech 22, 279 293

Murphy J, L'Estrange JL (1977) The performance and carcass fat characteristics of lambs fattened on concentrate diets. 1. Effect of maize and barley as the cereal source and of dietary supplementation with roughage, vitamin $E$, cobalt and vitamin $B_{12}$. Ir $J$ Agric Res 16, 187-204

Orskov ER, Duncan WR, Carnie CA (1975) Cereal processing and food utilization by sheep. III. Effect of replacement whole barley by whole oats on food utilization and firmness and composition of subcutaneous fat. Anim Prod 21, 51-59

Palmer DN, Husband DR, Winter PJ, Blunt JW, Jolly RD (1986) Ceroid lipofuscinosis in sheep. 1.bis (monoacylglycero) phosphate, dolichol, ubiquinone, phospholipids, fatty acid and fluorescence in liver lipopigment lipids. J Biol Chem 261, 1766-1772

Palmquist DL, Conrad HR (1980) High fat rations for dairy cows: tallow and hydrolysed blended fat at two intakes. J Dairy Sci 63, 391-395

Prache $S$, Aurousseau $B$, Thériez $M$, Renerre $M$ (1990) Les défauts de couleur du tissu adipeux sous-cutané des carcasses d'ovins. INRA Prod Anim 3, 275-285

Ray EE, Kromann RP, Cosma EJ (1975) Relationships between fatty acid composition of lamb fat and dietary components. J Anim Sci $41,1767-1774$

Robelin J, Thériez M, Arnal M, Ferrara M (1977) Évolution de la composition chimique de jeunes agneaux mâles jusqu'à l'âge de 16 semaines. Ann Zootech 26, 69-81

Snedecor GN, Cochran WG (1971) Méthodes statistiques. Association de coordination, Techniques Agricoles, Paris

Solomon MB, Lynch GP (1988) Biochemical, histochemical and palatability characteristics of young lambs affected by diet and electrical stimulation. J Anim Sci 65, 1955-1962

Sutton JD, Knight $R$, Mc Allan $A B$, Smith $R H$ (1983) Digestion and synthesis in the rumen of sheep given diets supplemented with free and protected oils. Br J Nutr 49, 419-432

Thériez M, Tissier M, Robelin J (1981) The chemical composition of the intensively fed lambs. Anim Prod 32, 29-37

Thieulin C (1986) Les divers facteurs influant sur l'utilisation digestive des matières grasses. Ann Nutr Alim 22, 245-258

Thompson JM, Parks JR (1985) Food intake, growth and body composition in Australian 
merino sheep selected for high and low weaning weight: 3. Energy balance. Anim Prod $40,85-91$

Turgeon OA, Brink DR, Bartle SJ, Klopfenstein TJ, Ferrell CL (1986) Effects of growth rate and compensatory growth on body composition in lambs. J Anim Sci 63, 770-780

Vezinhet A, Nougues J, Vigneron P (1976) Aspects généraux du développement et de la croissance des tissus musculaires et adipeux: caractéristiques chez les ovins. In :
Croissance, engraissement et qualité des carcasses. $2^{\text {es }}$ Journées Rech Ovine et Caprine INRA-ITOVIC, SPEOC, Paris, 27-43

Waghorn GC, Flux DS, Ulyatt MJ (1987) Effect of dietary protein and energy intakes on growth hormone, insulin, glucose tolerance and fatty acid synthesis in young wether sheep. Anim Prod 44, 143-152

Wahle KWJ, Garton GA (1972) Desaturation of stearic acid by sheep tissue microsomes. Proc Nutr Soc (abstr) 31, 110-A 